\title{
MPD Thruster Technology and Its Limitations
}

\section{Samarth Patel ${ }^{1 *}$, M.S.R. Bondugula ${ }^{2}$ and Srilochan Gorakula ${ }^{3}$}

\author{
${ }^{1}$ Aerospace Engineering, IIAEM, Jain (Deemed-to-be University), Bangalore, India \\ 2Space Engineering, Politecnico di Milano, Milan, Italy
}

${ }^{3}$ Aeronautical Engineering, Politecnico di Milano, Milan, Italy
E-mail: samarth1998.sp@gmail.com; marysharon.bondugula@mail.polimi.it; srilochan.gorakula@mail.polimi.it

*Corresponding author details: Samarth Patel; samarth1998.sp@gmail.com

\begin{abstract}
It was realized earlier that chemical propulsion systems utilize fuel very inefficiently, which greatly limits their lifespan. Electric propulsion is into existence to overcome this limitation of chemical propulsion. The magnetoplasmadynamic (MPD) thruster is presently the most powerful form of electromagnetic propulsion. It is the thruster's ability to efficiently convert MW of electric power into thrust which gives this technology a potential to perform several orbital as well as deep space missions. MPD thruster offers distinct advantages over conventional types of propulsion for several mission applications with its high specific impulse and exhaust velocities. However, MPD thruster has limitations which limits its operational efficiency and lifetime. In this paper, the thruster limitations are reviewed with respect to three operational limits i.e., the onset phenomenon, cathode lifetime, and thruster overfed limits. The dependence and effects of the operational limits on each other is compared using different empirical models to derive a scaling factor that has been found for each geometrical arrangement; a limiting value exists beyond which the operation becomes highly unsteady and electrode erosion occurs. Along with reviewing and proposing methods to overcome power limitations for MPD thrusters, the relation between exit velocity and ratio of electrode's radius is also verified using Maecker's formula.
\end{abstract}

Keywords: Magnetoplasmadynamic; Propulsion; Onset Phenomenon; Cathode Lifetime; Overfed

\section{INTRODUCTION}

Electric propulsion (EP) for spacecraft is defined as: the use of energy from a source not stored in the chemical bonds of the propellants to augment or provide the exhaust velocity of the propulsion device. Electric propulsion can be thought as an "end-run" around the limitations placed on our ability to increase the performance of rocket engines. The advantages of EP over chemical propulsion - nearly a factor of 10 reduction in propellant required to perform the same function [1]. 9 $\mathrm{kWh}$ is the minimum energy expenditure to take $1 \mathrm{~kg}$ of mass from the surface of the earth and place it in orbit about the earth. (To earth escape, twice this expenditure is required.) The maximum chemical energy that we can obtain per $\mathrm{kg}$ of $\mathrm{O}_{2}-\mathrm{H}_{2}$ propellant is only $3.7 \mathrm{kWh}$, so we can see that in placing chemical fuel in orbit for orbital transfer propulsion or for space missions, there is a huge energy expense. Chemical energy is inextricably linked to mass, so there is a hard-upper limit, around $500 \mathrm{~s}$, to the specific impulse that can be obtained from chemical rockets. The attractiveness of electrical propulsion is that propellant mass consumption can be greatly reduced because, in principle, any amount of electrical energy can be added to a given quantity of mass. To put it another way, the specific impulse has no hard-upper limit, and the energy source for electrical propulsion can be either accessible in space (solar energy) or brought from earth in the form of nuclear fuel whose energy density $(\mathrm{kWh} / \mathrm{kg})$ is orders of magnitude greater than that of chemical fuel [2][3].
In electric propulsion the term thruster is used the same way as engine is in liquid propellant and motor in solid propellant rockets. In addition to a separate energy source, such as solar or nuclear with its auxiliaries (concentrators, heat conductors, pumps, panels, and radiators), the basic subsystems of a typical space electric propulsion system are: (1) conversion devices to transform the spacecraft's electrical power to voltages, frequencies, pulse rates, and currents suitable for particular electrical propulsion systems; and (2) one or more thrusters to convert the electric energy into kinetic energy of the propellant exhaust [4][5]. Additionally, needed are: (3) a propellant system for storing, metering, and delivering the propellant and/or propellant fill provisions; (4) several controls for starting and stopping power and propellant flow; and some also need (5) thrust vector control units (also called TGAs—thrust/ gimbal assemblies).

Electromagnetic propulsion can be defined as acceleration achieved by the interaction of electric and magnetic fields within a plasma. Moderately dense plasmas, found in hightemperature and/or nonequilibrium gases, are electrically neutral overall and reasonably good conductors of electricity. Some devices add a nozzle to enhance performance [1][4][6]. Thrust in electromagnetic thrusters is generated through an interaction of crossed electric and magnetic fields. First, the propellant gas is ionised to produce a plasma. 
There are several ways to approach this but two of the more common are: using a high voltage to create an arc discharge; and using a radio frequency (RF) field to add energy to free electrons which ionize the gas via collisions. Once the plasma is established, current can flow and magnetic fields (either self-generated or applied) can react with the electric field present in the discharge to produce a force on the charged particles which is perpendicular to both the electric and magnetic fields.
High-power MPD thrusters are less developed than ion engines, and to a large extent are still laboratory items. Exploiting the Lorentz force, MPD acceleration occurs when a flow of charges, e.g., electrons and ions, in all respects equivalent to a current, J, moves in a magnetic field B. The Lorentz force is $\mathrm{F}=\mathrm{J} \times \mathrm{B}$ : it accelerates charged species moving in the magnetic field $\mathrm{B}$. The regime of an MPD thruster can be steady in the strict sense, or quasisteady. The thrust of a quasi-steady MPD may occur in pulses or bursts; when these last long enough, or when the burst repetition rate is high enough, the averaged thrust is said to be quasi-steady [7].

TABLE 1: SYNOPSIS OF THE COMPARISON BETWEEN DIFFERENT REGIONS OF APPLICATION OF SEVERAL ELECTRICAL PROPULSION SYSTEMS AND CHEMICAL PROPULSION SYSTEM IN TERMS OF POWER AND SPECIFIC IMPULSE.

\begin{tabular}{|l|c|c|c|c|}
\hline \multicolumn{1}{|c|}{ Type } & $\begin{array}{c}\text { Thrust } \\
\text { Range } \\
\text { (mN) }\end{array}$ & $\begin{array}{c}\text { Specific } \\
\text { Impulse } \\
\text { (sec) }\end{array}$ & $\begin{array}{c}\text { Thruster } \\
\text { Efficiency } \\
\text { (\%) }\end{array}$ & $\begin{array}{c}\text { Kinetic Power } \\
\text { per Unit Thrust } \\
\text { (w/mN) }\end{array}$ \\
\hline Resistojet (thermal) & $200-300$ & $200-350$ & $65-90$ & $05-6$ \\
\hline Arcjet (thermal) & $200-1000$ & $400-800$ & $30-50$ & $2-3$ \\
\hline Ion thruster & $0.01-500$ & $1500-8000$ & $60-80$ & $10-70$ \\
\hline Solid pulsed plasma (PPT) & $0.05-10$ & $600-2000$ & 10 & $10-50$ \\
\hline Magnetoplasmadynamic (MPD) & $0.001-2000$ & $2000-5000$ & $30-50$ & 100 \\
\hline Hall thruster & $0.01-2000$ & $1500-2000$ & $30-50$ & 100 \\
\hline Monopropellant rocket & $30-500,000$ & $200-250$ & $87-97$ & \\
\hline
\end{tabular}

It can be clearly observed that thrust levels of MPD thrusters are small relative to those of chemical rocket propulsion systems and that tiny valves for metering low flows are a challenge, but that values of specific impulse can be substantially higher; this translates into a longer operational life for those satellites whose life is propellant limited. Presently, electric thruster gives accelerations too low for overcoming the high-gravity fields of planetary launches.

MPDTs are an active topic of research because of their potential to offer both high thrust and high exhaust velocity. MPD thrusters have long held the promise of high exhaust velocity at MW power levels [8]. Magnetoplasmadynamic (MPD) thrusters operate on the principles of Electromagnetic propulsion along with Lorentz force (the force on a charged particle by an electromagnetic field) dealing with the acceleration of highly charged plasmas by the interaction of currents driven through the gas and magnetic fields created by those currents or by an external source to generate thrust. Various propellants such as xenon, neon, argon, hydrogen, hydrazine, and lithium have been used where lithium has the highest efficiency. An MPD thruster is also referred to as Lorentz Force Accelerator (LFA). According to Edgar Choueiri MPD thrusters have input power 100-500 kilowatts, exhaust velocity 15-60 kilometres per second, thrust 2.5-25 newtons and efficiency 40-60 percent along with additional research showing that exhaust velocities can exceed 100 kilometres per second [6],[9]. Electromagnetic propulsion exhibits a wide range of advantages over electrostatic propulsion like having a relatively high thrust density, which is about 10 to 100 times that of an ion engine and electromagnetic propulsion engine yields a natural exhaust beam eliminating the need for an ion neutraliser.

Electromagnetic propulsion and especially MPD thrusters have very specific and pertinent applications in the field of space exploration. MPD thrusters are currently being considered for applications as primary propulsion for both piloted and robotic and planetary missions [10].
This propulsion technique is used in overcoming translational and rotational perturbations in satellite orbits, increasing satellite speed while overcoming the relatively weak gravitational field some distance away from the earth, such as orbit raising, missions such as interplanetary travel and deep space probes are also candidates for electric propulsion and a number of newer missions look at this propulsion system for either precision attitude/position control or formation-flying relative position control needed for multi-satellite communications.

Although simple in design and robust in operation, MPD thruster performance has typically been limited by low thrust efficiency in regimes of interest. Severe electrode erosion has been observed at high values of specific impulse. This erosion is related to onset of significant discharge voltage oscillations and unsteady thruster operation Another limiting criterion is the thermo-ionic emission from the cathode. The erosion rate from the cathode determines its lifetime and hence the mission duration of the whole thruster. On the opposite direction, lower levels of current lead to unstable operation of the thruster and this state are known as the overfed state [11]. Some other factors are if the cathode is not properly sized the evaporation of cathode material leads to limited thruster life and it also depend on the type of operations i.e. Quasi steady operation and Steady operation. The onset phenomenon, cathode lifetime, and thruster overfed limits are then introduced as the main operating limits.

\section{DETAILED STUDY OF LIMITATIONS}

To study and analyse the MPD limitations, it is crucial to understand which kind of MPD thruster have we taken and what are the underlying assumptions.

\section{Self-Field MPD}

(William J. Coogan et al., 2016) studied that in an MPDT, there are three thrust generating mechanisms: Self-field (SF), Gas dynamic (GD), Applied-field (AF). There is a power regime for each component when that component 
is larger in comparison to the other two. For instance, if the $\mathrm{SF}$ thrust component is the major thrust component in SFMPDTs, and it is caused by the current's interaction with its own self-induced magnetic field. In AF-MPDTs, the SF thrust component is often dominating at high power [12].

FIGURE 1: SELF-FIELD MAGNETOPLASMADYNAMIC THRUSTER [13]

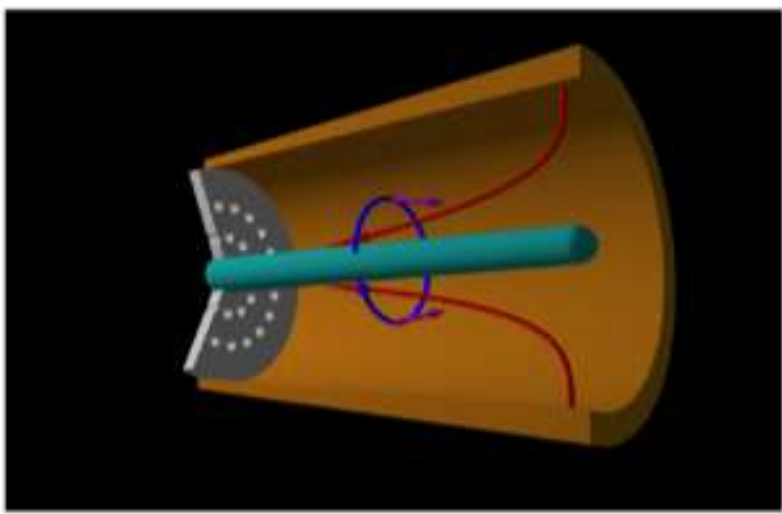

FIGURE 2: SIMPLIFIED DIAGRAM OF MPD THRUSTER OPERATION SHOWING CURRENT STREAMLINES AND SELF-INDUCED MAGNETIC FIELD.

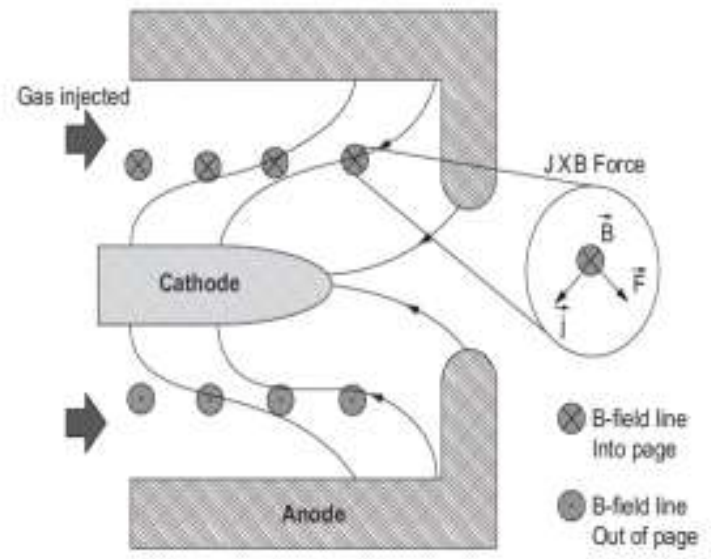

Brittany Lee Ilardi in their report found that current runs radially from the anode toward the cathode in a classic self-field MPDT, then axially down the cathode. An azimuthal magnetic field is produced by this current. The cross product of the azimuthal magnetic field and radial current generates Lorentz force in the axial direction, which in turn generates thrust. Fig. 1 depicts this event, where the current in red, the magnetic field in blue and the Lorentz force in purple. Despite its ability to generate exceptionally high thrust for electric propulsion, this technique demands a lot of work. To operate, a power range of 1 to $30 \mathrm{MW}$ is required. This is far beyond the ability of today's space-based power sources [13].

FIGURE 3: SELF FIELD PRINCIPLE OF OPERATION [14]

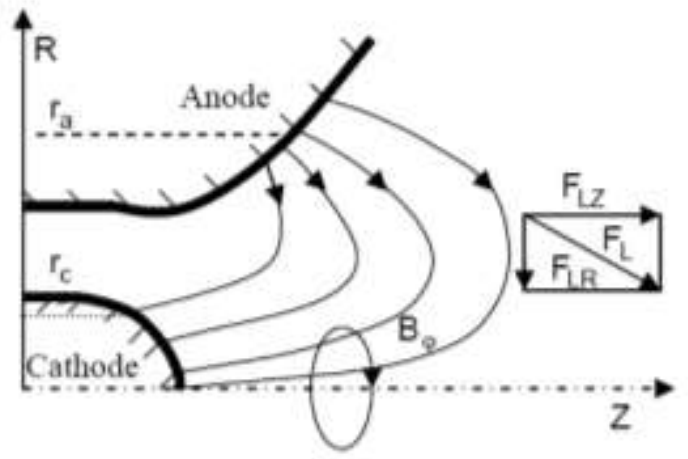

(O.A. Gorshkov et al., 2007) inferred in their paper about the working principle which is depicted in Fig.3, FLZ is directed along the thruster axis $\mathrm{Z}$ and accelerates plasma along this axis in this thruster electromagnetic force component. FLR is another component, which compresses plasma in the direction of the $\mathrm{Z}$ axis. Because it contributes to paraxial plasma heating and accelerates it along the $\mathrm{Z}$ axis, the latter force can be transformed into pushing force. When an external magnetic field is applied, the hall component force is produced by the interaction of the hall current with the radial component of the applied field [14]. (Carlos M. Xisto, et al., 2015) explained the working and limitations of MPDT. Due of their relatively poor efficiency for the relevant operational regimes, they are being used for space propulsion or satellite repositioning on a regular basis. For a particular impulse of 5500s and a power usage of $20 \mathrm{KW}$, the highest efficiency for an MPD nozzle was $69 \%$. Between 200 and $500 \mathrm{KW}$ is the most efficient operating range. When employing vaporized lithium as the propellant, the best efficiency $(50 \%)$ was recorded for a power value closer to $100 \mathrm{KW}$. However vaporized lithium usually causes condensation which might harm the space craft's surface. Hence, argon or hydrogen can be considered, although at the cost of lower efficiency. Also, the efficiency can be enhanced not just by choosing a propellant with superior electrical properties, but also by changing the electrode design or applying an externally generated magnetic field [15].

Jian Li et. al studied that the magnetic field is induced by the discharge current, which is a distinguishing property of self-field MPDT. The discharge current and induced magnetic field is coupled in the azimuthal direction to produce the magnetic force. The gas propellant is heated, ionized and accelerated as high-enthalpy plasma by the effect of electro thermal or electromagnetic exhaust at speeds of tens of kilometers per second. It's nearly impossible to enhance thruster performance without a thorough understanding of the process. This paper also inferred that the performance improvement of SF-MPDT is greatly restricted by low efficiency about 30\% [16].

\section{Applied-Field MPD}

Electric systems are efficient but have limited reactivity and manoeuvrability due to their limited thrust values. The Applied-Field Magnetoplasmadynamic (AF-MPD) Thruster generates thrust by combining magnetic and electric fields. AF-MPD thrusters have electromagnetic and gas-dynamic processes combined in a hybrid accelerator. Thrusters with a power range of 10 to $100 \mathrm{~kW}$ are the finest choice. These thrusters provide the flexibility and versatility required by modern space defence needs. AF-MPD thrusters accelerate plasma to high speeds and create high thrust density and specific impulse. Throttleability is a unique feature that refers to the ability to operate under various situations, including fluctuations in thrust. These thrusters operate on a few different concepts. The hall acceleration mechanism and azimuthal currents are two examples. Axial and radial Lorentz forces are produced by inducing azimuthal currents. The plasma is directly accelerated by the axial component, while the radial component builds up pressure. When applied meridional current crosses the applied magnetic field, rotational kinetic energy is formed in the plasma. Joule heating adds energy, and the fringing magnetic field converts it into axial jet kinetic energy.

\section{LIMITATIONS OF MPD THRUSTER}

Although simple in design and robust in operation, MPD thruster performance has typically been limited by low thrust efficiency in regimes of interest. 
The major limitations studied in MPDT are as follows:

- Thermo-ionic emission from cathode

- Onset phenomenon

- Overfed phenomenon

\section{Thermo-ionic emission from cathode}

The performance of MPD thrusters in interest domains was generally restricted by poor thrust efficiency [17]. Firstly, Cathode thermo-ionic emission is the key limiting criteria [18]. The cathode degradation rate affects its lifetime and consequently the whole thruster's endurance. Secondly, a well-known characteristic that the arc cathode attachment in a pulsed MPD thruster and at the beginning phase of a continuous MPD thruster resembles numerous hot spots of high current density [19][20]. These spots are often non-stationary and move over the cathode surface. Such a splotchy attachment is the most prevalent on cold metal cathodes of low-pressure arcs, and it appears to be an inherent characteristic of such discharges [21]. Further it can be interpreted that, the most critical features of a high current gas discharge with cold metal electrodes are arc cathode spots. Moreover, several theories had extensive study in determining the spot radius including research. Since the sequence analysis of electrical and optical measuring techniques was increased to the microsecond and even beyond the nanosecond range, scanning electron microscopy became a common technique to determine the arc traces left on cathode surfaces, much more has been learned about the mechanisms of this spotty attachment. The spots are distinguished by significant variations on a nanosecond time scale, as well as energy densities of up to $105 \mathrm{~J} / \mathrm{cm} 3$ or more and agile moments [22]. A spot is a collection of plasma and erosion craters. The craters replenish the discharge plasma with fresh ionized vapor. In a vacuum, this vapor is the only medium for the discharge to exist, but it is also a crucial component of a gaseous arc. As a result, the craters may be used to help comprehend the discharge, particularly because they are not just a passive witness to the arc, but also a required active component.

FIGURE 4: EVIDENCE FROM LIFE TEST THAT DISCHARGE CATHODE MUST BE PROTECTED FROM ION SPUTTERING. (A) PRE-TEST DISCHARGE CATHODE. (B) POST-TEST DISCHARGE CATHODE. (IMAGE COURTESY OF NASA)

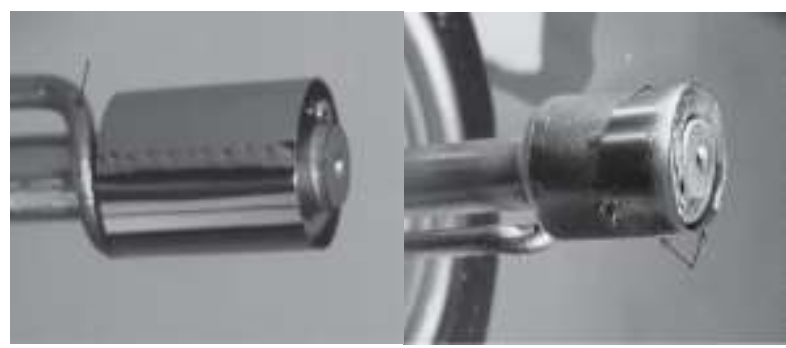

Furthermore, a significant research has been done to distinguish between micro spots with diameters of several micrometres with displacement velocities of several hundred meters per second, and spots that are much more stationary with diameters of up to 100 nanometres. These additional, bigger spots appear to have been the consequence of the spot cluster, that is the micro spots are not distributed over a broader area but cling to smaller areas owing to favorable emission circumstances, and local overheating create larger melted areas, as well as rather significant cathode damage [23]

Moreover, in depth study has done on the temperature effect on the cathode. The high erosive extraction is typical of cold cathode operation, while thermionic current emission occurs in heated-cathode operation that substantially lowers the rate of erosion of electrode [24]. However, the major benefit of cathode heating is the substantial drop in the erosion rate, which indicates directly lengthening thruster life [25]. To sum up, the cathode erosion has a pivotal role in the thruster lifetime and many factors can contribute to it.

\section{Onset phenomenon}

The onset phenomenon is the primary constraint on MPD thruster performance. Near this limit, the thruster will have the maximum specific impulse, efficiency, and thrust. The pumping component causes charge carriers to move towards the thruster axis and deplete or decrease these carriers near the anode at high currents. As a result, the plasma resistance rises, and the discharge voltage rises for the same current level. A critical current with a fixed mass flow and geometry relates to increased erosion of thruster components (electrode ablation) and large voltage oscillations (induced by plasma turbulence, lack of appropriate frequency and power density) [26].

The onset is caused by a decrease in particle density near the anode, primarily caused by the Hall effect and correlated with the Lorentz-force component derived from the interaction of the axial component of current density with the azimuthal component of the self-induced magnetic field. As a result, any increase in anode-adjacent particle density should postpone anode starvation. As a result, any increase in anode-adjacent particle density caused by propellant species or geometry changes should delay anode starvation [27]. The onset value is used to calculate the mass flow rate of the propellant. When insufficient charge carriers in the near anode region support the required current pattern, onset occurs. When the electrode lengths are roughly equal, the performance is optimal. The cathode radius and anode to cathode ratio are varied with current fluctuations to understand the consequences of geometry change.

The increase in terminal voltage caused by the back emf $\mathrm{u}$ $\mathrm{x}$ B) associated with a self-magnetic field at the cathode indicates intended electromagnetic acceleration. At the onset current, a rapid increase in terminal voltage occurs, dependent on propellant injection geometry [28]. When the propellant is injected at the cathode radius, the terminal voltage rises, and the anode fall voltage rises. The increase in radius is linked to back emf in the near cathode plasma. Because the oscillations have no effect on the discharge geometry at currents below onset, the terminal voltage is unaffected.

According to some authors, the emf is responsible for lowering the plasma's effective electric field in the central part of the acceleration channel and the electrode current attachment zone. It is also claimed that plasma might move fast enough to obstruct current flow between electrodes. These theories are deficient in a few areas because they focus on specific configurations or operational conditions rather than the underlying cause of onset in the broadest sense. None of the ideas account for the appearance of the voltage hash or the patchy current attachment close or beyond the beginning [27],[28]. The existence of anode spots is sometimes accepted without attempting to explain their origin, and the voltage hash is then explained as the result of anode spot generation, extinction, and mobility.

Recently, a theory has surfaced concerning discussions about spot generation. It can result from filamentation instability, a plasma instability that causes current to fragment into multiple channels regardless of the anode material. Current filamentation looks a lot like the anode spots phenomenon, and it has been seen in other plasmapinch devices similar to MPD thrusters. As a result, the 
filamentation technique could be a potential indication of the onset's understanding.

\section{Overfed phenomenon}

Nada inferred that the primary operating restrictions of a working Magnetoplasmadynamic Thruster is the onset phenomenon, cathode lifetime and the overfed limits. When the charge carrier moves toward the thruster axis, depletion of these carriers near the anode at high currents is observed. This causes the plasma resistance to rise for the same current levels. In these conditions, extreme fluctuations in discharge voltage and electrode ablation are observed. This is called as Onset phenomenon. Increased degradation of thruster components and huge voltage fluctuations at a critical current with a fixed mass flow and geometry are all part of this process. Cathode's lifetime and in turn the thruster's overall mission duration is determined by its rate of the depletion of cathode. On the opposite direction, lower current levels cause the thruster to operate in an unsteady manner. This is known as the overfed state.

The thruster is discovered to be 'overfed' below a particular amount of $\mathrm{J}^{2} / \mathrm{m}$ (Choueiri proves that the $\left(\mathrm{m}^{*}\right.$ ) is a universal mass flow rate and equals $66 \mathrm{~g} / \mathrm{s}$. J is the current [9]) and it endures erosive conditions. This happens because the current level is low and/or the mass flow rate is large. Lower levels of current means fewer electrons released. However, a high mass flow rate means dense plasma within the discharge path. The plasma plume is quite erratic under these conditions and the cathode has an orange/yellow color and does not generate electron efficiently. (Myers et al., 1991) suggested that overfed regime occurs at $\mathrm{J}^{2} / \mathrm{m} \leq 2 \times 10^{10}\left(\mathrm{~A}^{2} \mathrm{~s} \mathrm{~kg}^{-1}\right)$, while Wood et al measured it at $\mathrm{J}^{2} / \mathrm{m} \leq 2 \cdot 7 \times 10^{10}\left(\mathrm{~A}^{2} \mathrm{~s} \mathrm{~kg}^{-1}\right)$.

\section{FIGURE 5: OPERATING LIMITS OF MPDT}

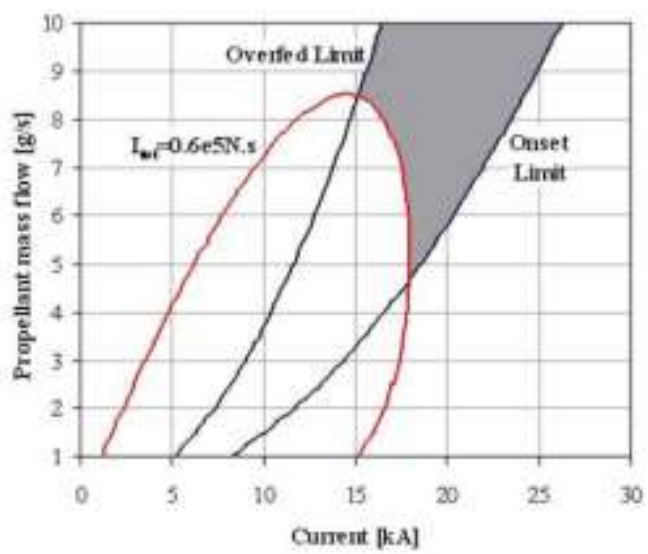

The line depicting overfed limit is shown in Fig.5 employing $\mathrm{J}^{2} / \mathrm{m} .=2 \cdot 7 \times 1010\left(\mathrm{~A}^{2} \mathrm{~s} \mathrm{~kg}^{-1}\right)$. For a thruster with argon as the propellant, cathode and anode lengths of $10 \mathrm{~cm}$, cathode radius of $0.95 \mathrm{~cm}$, and anode to cathode radius ratio of 5 is obtained. The curve is plotted to represent the total impulse of ( $\left.\mathrm{I}_{\text {tot }}=0.6 \mathrm{e} 5 \mathrm{~N} . \mathrm{s}\right)$.

The operational limits of the thruster are depicted by the three curves from Fig.5. if a mission with at least ( $\mathrm{I}_{\text {tot }}=$ $0 \cdot 6 \mathrm{e} 5 \mathrm{~N} . \mathrm{s}$ ) is considered, the shaded area to the left of the onset limit, to the right of the overfed limit and outside the total impulse contour reflects the safe and adequate regime of operation for the thruster. The overfed limit does not impede the performance for the investigated range and current and mass flow rate as shown in Fig.5.

The impacts of the MPDT operating limits such as the overfed state, onset phenomenon and the cathode lifetime on the optimization for certain missions are not published [10]. The research on overfed state is very limited.

\section{PHYSICS OF MPDT}

To further study and understand the Onset and Overfed phenomenon along with its effect on Cathode erosion, we propose to derive a scaling factor from the generalized empirical model of Maecker, popularly known as Maecker's formula for each geometrical arrangement; a limiting value that must exists beyond which the operation becomes highly unsteady and electrode erosion occurs.

\section{Electromagnetic forces}

To begin with Lorentz Force; if charge $\mathrm{q}$ is moving at velocity $\vec{v}$, in an electric field $\vec{E}$ and in magnetic field $\vec{B}$, then the Lorentz force can be given as,

$$
\vec{F}=q(\vec{E}+\vec{v} \times \vec{B})
$$

In equation (1) only $\vec{E}$, must be different in different reference frames. Let $\vec{E}$ be the electric filed in laboratory frame and $\vec{E}^{\prime}$ in another frame moving at velocity $\vec{u}$, relative to first one. Thus,

$\vec{E}+\vec{v} \times \vec{B}=\vec{E}^{\prime}+(\vec{v}-\vec{u}) \times \vec{B}$

$\left.\therefore \vec{E}^{\prime}=\overrightarrow{(E}+\vec{u} \times \vec{B}\right)$

If $\vec{u}=\vec{v}$, the Lorentz force is purely electrostatic

$$
\text { i.e. } \vec{F}_{\text {Lor }}=\mathrm{q} \vec{E}^{\prime}
$$

The frame moving at $\vec{u}$ velocity is often chosen to be the one moving at the mean mass velocity of the plasma. Now consider a plasma having a number density $\mathrm{n}_{\mathrm{j}}$ of the $\mathrm{j}^{\text {th }}$ type of charged particles, which have a charge $\mathrm{q}_{\mathrm{j}} \&$ move with velocity $\vec{v}_{\text {j. }}$.

Hence, the net Lorentz force per unit volume is given by,

$\left.\vec{f}=\Sigma_{j} n_{j} q_{j} \overrightarrow{(E}+\vec{v} \times \vec{B}\right)$

And since the plasma is neutral, we have,

$\Sigma_{j} n_{j} q_{j}=0$

Putting equation (5) in equation (4), we get

$\vec{f}=\Sigma_{j}\left(n_{j} q_{j} \overrightarrow{v_{j}}\right) \times \vec{B}$

But we also know that,

$\Sigma_{j}\left(n_{j} q_{j} \overrightarrow{\left.v_{j}\right)}=\vec{j}\right.$

Where $\vec{j}$ is Current Density vector $\left(\mathrm{A} / \mathrm{m}^{2}\right)$

Finally, we get,

$\vec{f}=\vec{j} \times \vec{B}$

Simple plasma accelerator OR an ideal MPDT Let us take a Simple Plasma Accelerator OR an Ideal MPDT:

Plasma flowing in at velocity $\vec{u}$ and an external electric field $\vec{E}$ applied to it. A current,

$$
\vec{J}=\sigma \vec{E}^{r}=\sigma(\vec{E}+\vec{v} \times \vec{B})
$$

will flow in direction of $\vec{E}$. Where,

$\sigma=$ scalar conductivity (quantifies how well a material conducts electricity) 


$$
\sigma=\frac{e^{2} n_{e}}{m_{e} v_{e}}
$$

Where, e stands for electron as they provide dominant contribution to $\vec{j}$.

\section{Basic design of MPDT}

Now let us consider a basic design of MPDT

\section{Calculation of thrust:}

Total thrust has 2 contributions

1) Integral of gas pressure over the back facing surface Electrothermal or Aerodynamic Thrust. (It will be present only if joule heating effect dominates over the Lorentz Work)

2) Reaction to the Lorentz forces exerted on the plasma.

At high efficiencies, the electromagnetic thrust dominates over the electrothermal thrust.

$$
\begin{aligned}
& \vec{F}_{\text {Ем }}=\int \vec{J} \times \vec{B} d V \\
& =\frac{1}{\mu_{0}} \int(\nabla \times \vec{B}) \times \vec{B} d V
\end{aligned}
$$

Where $\mu_{0}=4 \pi \times 10^{-7} \frac{\mathrm{H}}{\mathrm{m}}=$ Magnetic Permeability of Vacuum.

Now in general

$$
(\nabla \times \vec{B}) \times \vec{B}=(\vec{B} \cdot \nabla) \vec{B}-\nabla\left(\frac{B^{2}}{2}\right)
$$

But since $|\vec{B}|$ does not vary along its own direction in our case,

$$
(\vec{B} \cdot \nabla) \vec{B}=-\frac{B^{2}}{r} \hat{l}_{r}
$$

( $\because$ No axial component, integrates to zero by symmetry)

$$
\vec{F}_{\text {Ем }}=-\frac{1}{\mu_{0}} \int \nabla\left(\frac{B^{2}}{2}\right) \mathrm{dV}
$$

Now by vectorial version of Gauss's Theorem We get,

$$
\vec{F}_{\text {EM }}=-\frac{1}{\mu_{0}} \int\left(\frac{B^{2}}{2}\right) \mathrm{d} \vec{A}
$$

Where integral extends to the surface surrounding the plasma \& $\mathrm{d} \vec{A}$ points outwards from that surface. However, we are only interested in the axial force. So,

$$
\vec{F}_{\text {EM }}=-\frac{1}{\mu_{0}} \int\left(\frac{B^{2}}{2}\right) \mathrm{dA}
$$

$\left(d A_{x}\right.$ is the projection of each area element normal to the axial direction)

Particularly for any cylindrical surface $d A_{x}=0$. The only surface surrounding the plasma are the back plate, the cathode tip \& anode rim.

For backplate, using equation (5) with $\mathrm{I}^{\prime}=\mathrm{I}$, we calculate the contribution.

$$
\begin{aligned}
\mathrm{F}_{\mathrm{EM}} \text { backplate }= & +\frac{1}{\mu_{0}} \int_{R e}^{R_{a}} \frac{1}{2}\left(\frac{\mu_{0} I}{2 \pi r}\right) 2 \pi r \mathrm{dr} \\
& =\mu_{0} \frac{I^{2}}{4 \pi} \ln \frac{R_{a}}{\mathrm{Rc}}
\end{aligned}
$$

Where $R_{a}$ and $R_{c}$ are the anode and cathode radii and $(+)$ sign is because the normal $\mathrm{dA}$ to the surface, points backwards (i.e. out of the plasma)

Maecker's formula:

We have,

$$
\mathrm{F} \approx \mathrm{F}_{\mathrm{EM}} \text { backplate }
$$

[also known as Maecker's law/formula]

$$
\therefore \mathrm{T}=\frac{\mu_{0} I^{2}}{4 \pi} \ln \frac{R a}{R e} I^{2}
$$

[I is the thruster current]

It can also be written as,

$$
\mathrm{T}=\frac{1}{2} L^{\prime} I^{2}
$$

Where, $L^{\prime}$ is the channel inductance per unit length.

Now in a more complex channel geometry and taking into account finite cathode length and pressure effects on the cathode tip, the above expression can be generalized with the inclusion of a correction term.

$$
\mathrm{T}=\frac{\mu_{0} I^{2}}{4 \pi} \ln \left(\frac{R_{a}}{\mathrm{Re}}+\mathrm{A}\right)
$$

For instance, in the case of a conical cathode tip one would find $\mathrm{A}=3 / 4$.

Using equation (9), the expression for exit velocity is

$$
u_{e}=\frac{F}{M} \approx\left(\frac{\mu_{0}}{4 \pi} \ln \frac{R a}{R e}\right) \frac{I^{2}}{\mathrm{~m}}
$$

\section{Performance scaling factor:}

The speed of sound of representative points in the flow will scale like $M^{-1}$ ( $\mathrm{M}$ is the molecular mass of gas) and from equation (12) we see that Mach number must vary like the quantity,

$$
\frac{I^{2} \sqrt{M}}{\mathrm{~m}}
$$

This has been found out the most important actor for the scaling parameters for the MPD thrusters. It has been found that for each geometric arrangement, a limiting value $\frac{I^{2} \sqrt{M}}{\mathrm{~m}}$ exists, beyond which the operation becomes highly unsteady, and the erosion of electrodes increases.

\section{POWER LIMITATIONS}

One major issue is that optimal performance requires power requirements in the hundreds of kilowatts range. Interplanetary spacecraft power systems (such as radioisotope thermoelectric generators and solar arrays) are currently incapable of generating that much power. Commercial interest is low due to the power requirements in the order of hundreds of kilowatts. In 2005, NASA's Project Prometheus (use of a small nuclear reactor) reactor was expected to generate power in the hundreds of KW range but was failed to do that [29][30][31].

As they tend to have higher power requirements. Therefore, they have not been used as a dedicated thruster on operational spacecraft to date, although they've been researched since the 1960s in the USSR, the USA, Western Germany, Italy, and Japan. Only a few demonstrators have flown on both Russian and Japanese experimental satellites. They remain an attractive and cost-efficient option for high-thrust electric propulsion including Mars transfer engines. If MPD results in poor performance if operated below the levels of $100 \mathrm{~kW}$ [31][32]. 
In Applied field, which is low and medium powered MPD's require around $30-500 \mathrm{~kW}$ of electric power. The advantage of applied field MPD thrusters over a self-field type are that the magnetic fields can be manipulated independent of the amount of charge running through the cathode and anode, which can mean longer component life for the thruster [33][34][35][36]. In self-field, high powered MPD's require power around 1.5 MW which is a very difficult requirement to meet in space except for nuclear reactors whose development is stagnant. The stronger the current is, the stronger the magnetic field will be, and, in turn, the Lorentz acceleration which says the current depends on the input electric power [37].

Advantages of self-field are: reduces complications arising from using an external magnet, and provides good efficiencies and attractive performance parameters. At sufficient power, you can get away without coil or magnets, making the system lighter, simpler, and less temperaturesensitive. This is why most very high-powered systems use the self-field type of MPD. The performance possibilities have many people considering the technology as a key technology for rapid, man rated interplanetary transport, in particular to Mars [29][38].

\section{Power Deposition:}

The single largest power loss mechanism in MPD thrusters operated at power levels below 2-3MW is anode power deposition. The power deposited into the anode is given by [39][40][41],

$$
\mathrm{P}_{\mathrm{a}}=\mathrm{J}\left(\mathrm{V}_{\mathrm{a}}+\frac{5}{2} \frac{K T e}{e}+\emptyset\right)+\mathrm{P}_{\text {conv }}+\mathrm{P}_{\mathrm{rad}}
$$

First terms - current conduction into the anode $\mathrm{P}_{\text {conv }}$ - convective term due to hot plasma flow $\mathrm{P}_{\mathrm{rad}}$ - radiation from the plasma

For low power steady state thrusters, $80 \%$ input power deposited into the anode \& $20 \%$ for plasma heating and acceleration [41]. Techniques for reducing the anode power fraction is injecting propellant near the anode surface \& applying magnetic fields. Former technique increases the number of current carriers in the anode region \& is thought to decrease Va; while adding the magnetic field increases the total power deposited into the thruster and decreases the fraction of that power deposited into the anode. Anode power fraction is heavily dependent on mass flow rate and exhaust velocity [42][43].

Propellant - Argon
\begin{tabular}{|c|c|}
\hline Anode Power Fraction & Power \\
\hline $50 \%$ & $1.9 \mathrm{MW}$ \\
\hline $23 \%$ & $7 \mathrm{MW}$ \\
\hline
\end{tabular}

Propellant - Helium

\begin{tabular}{|c|c|}
\hline Anode Power Fraction & Power \\
\hline $70 \%$ & $150 \mathrm{~kW}$ \\
\hline $25 \%$ & $3.2 \mathrm{MW}$ \\
\hline
\end{tabular}

From the above tables, we can see the APF percentage decreases with decrease in mass flow rate and increase in thruster power. There is a substantial evidence for thrusters operated on argon propellant that the plasma is nearly fully ionized for most operating conditions [43] [44].

Methods to overcome the power losses are by increasing applied field strength from $0.01 \mathrm{~T}$ to $0.15 \mathrm{~T}$ decreased the anode power fraction from $80 \%$ to $60 \%$ for the thruster geometry and magnetic field increases power deposited into the plasma more rapidly than it deposits power into the anode. Cathode is not a significant power sink in steady state thrusters, though its importance in quasi steady state is poorly understood. In steady state thrusters - cathode consumes between $5 \%$ and $15 \%$ of the input power depending on thruster geometry and operating condition. No direct measurements exist for quasi-steady thrusters cathode fall voltage (cathode power deposition) is significantly higher for pulsed devices [43][44].

Other power loss mechanisms: Propellant ionization (argon - power sink of $3.8 * 10^{5} \mathrm{~W} / \mathrm{kg} / \mathrm{s}$ of flow rate). Heat transferred to rear chamber wall - dominant heat source is radiation from cathode surface, which is limited by viewfactor considerations to less than one-half the cathode power. Unrecovered thermal energy - vary widely due to the large uncertainty in the ion temperature which may be between 5000 and 100,000 K [44].

\section{PROPELLANT SELECTION FOR MPDT}

The propellant for chemical propulsion is hydrazine $\left(\mathrm{N}_{2} \mathrm{H}_{4}\right)$ and is commonly used on satellites and spacecraft. For electromagnetic thrusters, propellant selection is more about ion production cost and charge-to-mass ratio. This facilitates high thruster efficiency. A high propellant molecular mass facilitates operation at high propellant utilization efficiency and if specific impulse is held at its optimal value, it assures operation at a high net acceleration voltage, $V_{N}$, where thruster electrical efficiency is high.

Achievement of this criterion is also aided by low first ionization and high first excitation potentials. Such potentials facilitate minimal unwanted radiation losses and a low ion production cost. Another important operating feature is a high thrust-to-power ratio. High molecular mass propellants also help to achieve this. High thrust-to-power is important because most EP systems are power constrained and maximizing thrust keeps trip times (or maneuver durations) shorter. Additionally, monoatomic propellant species are preferred because they ionize predictably, and their expulsion is easily measured onboard the spacecraft to allow for operational controllability. Propellants with a low first ionization potential and a high second ionization potential are favored because doubly charged ions and charge exchange ions cause excessive erosion of the thruster surfaces. This is one of the main contributors to thruster wear out mechanisms that limit lifetime.

TABLE 2: PROPELLANTS MOST FAVORABLE FOR ELECTROSTATIC AND ELECTROMAGNETIC THRUSTERS

\begin{tabular}{|c|c|c|c|}
\hline $\begin{array}{c}\text { Propellan } \\
\mathbf{t}\end{array}$ & $\begin{array}{c}\text { Molecula } \\
\text { r Weight }\end{array}$ & $\begin{array}{c}\text { Storabilit } \\
\mathbf{y}\end{array}$ & $\begin{array}{c}\text { Compatibilit } \\
\mathbf{y}\end{array}$ \\
\hline Cesium & 133 & Good & Poor \\
\hline Mercury & 201 & Very good & Good \\
\hline Xenon & 131 & Very good & Excellent \\
\hline krypton & 84 & Good & Excellent \\
\hline Iodine & 254 & Excellent & Poor \\
\hline
\end{tabular}

\section{RESULTS AND DISCUSSIONS}

The aim to fully understand the limitations satisfies when we have a scaling factor which acts as the most important performance parameter as it gives the relation between Current I and the Onset-Overfed phenomenon. The cathode erosion can be estimated in the same regard as the inter-relation between Onset phenomenon, Overfed phenomenon and Cathode erosion is established. It has been found out the most important actor for the scaling parameters for the MPD thrusters. It has been found that for each geometric arrangement, a limiting value $\frac{I^{2} \sqrt{M}}{\mathrm{~m}}$ 
exists, beyond which the operation becomes highly unsteady, and the erosion of electrodes increases. Another important relation derived was between the Current I and the ratio of Anode radius to the Cathode radius. It helps in knowing how the electrode geometry $\left(R_{a} \& R_{c}\right)$ affects the Current I, which will allow the designer to set such radii that the value of Current I is optimal to avoid the instability in the operation as well as Cathode erosion.

\section{FIGURE 6: VARIATION OF CURRENT WITH RESPECT TO THE RATIO OF ANODE RADIUS TO CATHODE RADIUS USING MAECKER'S EMPIRICAL MODEL}

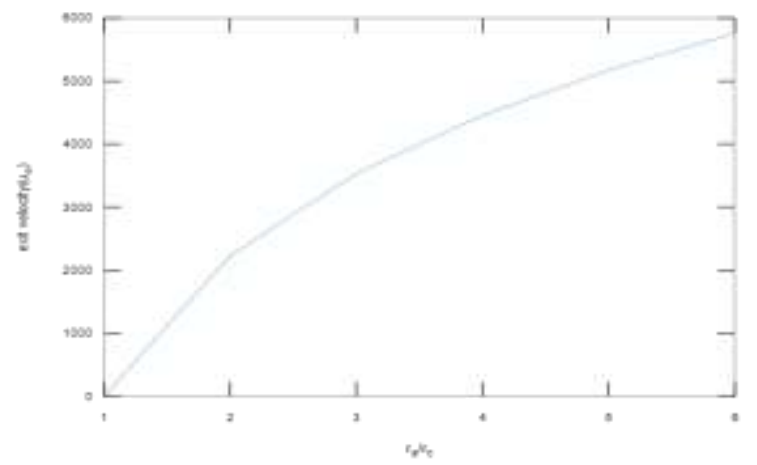

CONCLUSIONS AND FUTURE SCOPE

In conclusion, the space propulsion technology described above provides insight into some of the physical processes occurring in the MPD thruster. With all the understandings of the need and potential application of MPD thruster in space exploration, it is derived that there are still lots of limitations to overcome. Limitations which are restricting us to a lesser efficient model and to a comparatively smaller lifespan of the thruster. The Onset and Overfed phenomenon are the challenges to be solved to overcome the former, while Cathode erosion must be brought to a stable and minimised stage to overcome the latter. To do so, this study clarifies and verifies the limitations to its depth with the help of the most generalised empirical model. The inter-relation between Onset, Overfed and Cathode erosion is derived to be relying on the value of current I provided to the thruster using Maecker's formula Finally, along with the three major limitations, several factors such as power limitations and the importance of propellant selection also plays a role in the overall efficiency of the MPDT.

\section{ACKNOWLEDGMENT}

"Great things are not done by one person. They're done by a team of people." - Steve Jobs

This paper becomes a reality with the kind support and help of many individuals. we would like to extend our sincere thanks to all of them.

Foremost, we want to offer this endeavor to our GOD Almighty for the wisdom he bestowed upon us, the strength, peace of mind and good health to finish this research. Furthermore, we would like to thank our families and friends without whose support and encouragement this would have been impossible for us to undertake.

\section{REFERENCES}

[1] Stephen D. Heister, William E. Anderson, Timothée L. Pourpoint, R. Joseph Cassady. (2019). Rocket Propulsion. Cambridge University Press.

[2] Philip G. Hill \& Carl R. Peterson. (1965). Mechanics and Thermodynamics of Propulsion.
[3] R. Albertoni, F. Paganucci \& M. Andrenuccic. (2014). A phenomenological performance model for applied field MPD thrusters.

[4] Vatsal Sheth. Spacecraft Electric Propulsion - A review. September (2014). INTERNATIONAL JOURNAL OF RESEARCH IN AERONAUTICAL AND MECHANICAL ENGINEERING. Vol.2 Issue.9, Pgs: 43-55.

[5] Miroslaw Adamski (2017) Analysis of propulsion systems of unmanned aerial vehicles, Journal of Marine Engineering \& Technology, 16:4, 291-297, DOI: $10.1080 / 20464177.2017 .1383337$

[6] George P. Sutton \& Oscar Biblarz. (2017). Rocket Propulsion Elements - Ninth Edition.

[7] Paul A. Czysz \& Claudio Bruno. (2001). Future Spacecraft Propulsion Systems - Enabling Technologies for Space Exploration.

[8] James Gilland and Geoffrey Johnston. (2003). MPD Thruster Performance Analytic Models.

[9] Edgar Choueiri*. (1998). Scaling of Thrust in SelfField Magnetoplasmadynamic Thrusters.

[10] Roger M Myers, Maris A Mantenieks \& Michael R LaPointe. (1991). MPD thruster technology.

[11] T. R. Nada. (2016). Performance characterisation of MPD thrusters.

[12] William J. Coogan, Michael A. Hepler and Edgar Y. Choueiri. (2016). Measurement of the Applied-Field Component of the Thrust of a Lithium Lorentz Force Accelerator, Princeton University, Princeton, NJ, 08544, USA.

[13] Professor Edgar Choueiri. (28 April 2016). Control and Analysis of a JxB Pump Propellant Feed System for the Lithium Lorentz Force Accelerator, Brittany Lee Ilardi, Department of Mechanical and Aerospace Engineering, Princeton University in fulfilment of the requirements for, Undergraduate Independent Work, Final Report.

[14] O.A. Gorshkov*, V.N. Shutov**. (September 17-20, 2007). Development of High Power Magnetoplasmadynamic Thrusters in the USSR, IEPC-2007-136, Presented at the 30th International Electric Propulsion Conference, Florence,Italy. Keldysh Research Center, Moscow, 125438, Russia, K.N. Kozubsky, EDB “Fakel”, Kaliningrad, Russia, V.G. Ostrovsky, RSC "Energia”, 141070, Russia, V.A. Obukhov, RIAME MAI, Moscow, 125871, Russia.

[15] Carlos M. Xisto n, José C. Páscoa, Paulo J. Oliveira. (2015). Numerical analysis of real gas MHD flow on two-dimensional self-field MPD thrusters.

[16] Jian Li *, Yu Zhang, Jianjun $\mathrm{Wu}^{*}$, Yuqiang Cheng and Xinru Du. (2019). Particle Simulation Model for SelfField Magneto plasma dynamicThruster.

[17] FRUCHTMAN, A. (May 2003, 10). Limits on the efficiency of several electric thruster configurations, Physics of Plasma.

[18] MYERS, R.M., SUZUKI, N., KELLY, A.J. and JAHN, R.G. (1991). Cathode phenomena in low power magnetoplasmadynamic thruster, J Propul and Power, 7, (51), pp 760-766. 
[19] Hugel, H. and Kru'lle, G. (1969). "Phanomenologie and Energiebilanz von Lichtbogenkathoden bei Niedrigen Drucken und Hohen Stromstarken," Beitrdge der Plasmaphysik, Vol. 9, p. 87. 5

[20] Schrade, H. O., Auweter-Kurtz, M., and Kurtz, H. L., (April 1985). "Plasma Thruster Development," Institut fur Raumfahrtantriebe, Universitat Stuttgart, Rept. IRA 85-P3.

[21] Guile, A. E. and Juttner, B. (1980). "Basic Erosion Processes of Oxidized and Clean Metal Cathodes by Electric Arcs," IEEE Transactions on Plasma Science, Vol. PS-8, No. 3, p. 259.

[22] G. Ecker, Ergeb. Naturwiss, vol. 33, p. 1, 1961; Beitr. Plasmaphys., vol. 11, p. 405, 1971; IEEE Trans. (July 1979). Plasma Sci., vol. PS-4, p. 218, 1976; Forschungsber. Ruhr-Universitat Bochum, 79-02- 001.

[23] Bushik, A. I., Juttner, B., Pursch, H., and Shilov, A. (Feb 1983). "Effects of Local Heat Accumulation at the Cathode of Vacuum Arcs," Preprint: Akademie der Wissenschaften der DDR Zentralinstitut fur Elektronenphysik.

[24] H.O. Schrade, M. Auweter Kurtz and H.L. Kurtz. (1985). Cathode Erosion Studies on MPD Thrusters", AIAA-85-2019. 18th International Electric Propulsion Conference, Alexandria, Virginia.

[25] J. E. Polk, A. J. Kelly, R. G. Jahn. (1988). "Characterization of Cold Cathode Erosion Processes", IEPC-88-075, 20th International Electric Propulsion Conference, Garmish Partenkirchen, W.Germany.

[26] T. R. Nada. (2016). Performance characterisation of MPD thrusters.

[27] Mariano Andrenucci. (Dec 2010). Alternative Propulsion.

[28] J.W. Barnett and R.G. Jahn Onset. (1985). Phenomenon in MPD Thrusters.

[29] Nada, Tarek. (2007). Performance characterisation of MPD thrusters. Aeronautical Journal. 111. 443452. 10.1017/S000192400000470X.

[30] Choueiri., (1997). On the Thrust of Self-Field MPD Thrusters,Choueiri https://alfven.princeton.edu/publications/choueiriiepc-1997-121

[31] Myers Sverdup. (1991). Applied-Field MPD Thruster Geometry Effects, https://ntrs.nasa.gov/archive/nasa/casi.ntrs.nasa.g ov/19910017903.pdf

[32] Paganucci et al., (2001). Performance of an Applied Field MPDThruster, http://erps.spacegrant.org/uploads/images/images /iepc_articledownload_19882007/2001index/2002iepc/papers/t12/132_2.pdf.
[33] Mayer, T., Gabrielli, R. A., Boxberger, A., Herdrich, G., and Petkow, D. (September 2013). "Development of Analytical Scaling Models for Applied Field Magnetoplasmadynamic Thrusters," 64th International Astronautical Congress, International Astronautical Federation, Beijing.

[34] Myers, R. M., (1995). "Geometric Scaling of AppliedField Magnetoplasmadynamic Thrusters,” Journal of Propulsion and Power, Vol. 11, No. 2, pages 343-350.

[35] Tikhonov, V. B., Semenikhin S. A., Brophy J.R., and Polk J.E., (1997). "Performance of $130 \mathrm{~kW}$ MPD Thruster with an External Magnetic Field and Li as a Propellant", International Electric Propulsion Conference, IEPC 97117, Cleveland, Ohio, pp. 728-733.

[36] Boxberger, A., et al. (2012). "Experimental Investigation of Steady-State Applied-Field Magnetoplasmadynamic Thrusters at Institute of Space Systems", 48th AIAA/ASME/SAE/ASEE Joint Propulsion Conference \& Exhibit, Atlanta, Georgia.

[37] Boxberger, A., and G. Herdrich. (2017). "Integral Measurements of $100 \mathrm{~kW}$ Class Steady State AppliedField Magnetoplasmadynamic Thruster SX3 and Perspectives of AF-MPD Technology." 35th International Electric Propulsion Conference.

[38] Barnett J. W. (April 1985). "Operation of the MPD Thruster with Stepped Current Input," Ph.D. Thesis, Dept. of Mech. and Aero. Engineering, Princeton University.

[39] Preble, J.C. (May 1990). "Onset in Magnetoplasmadynamic Thrusters: A Model of an Electrothermal Instability," M.S. Thesis, Dept. of Aero. and Astro., Massachussets Inst. of Technology.

[40] Gallimore, A. D., Kelly, A. J., and Jahn, R. G. (July 1990). "Anode Power Deposition in Quasi-Steady MPD Thrusters, "AIAA 90-2668.

[41] Gallimore, A. D., Myers, R. M., Kelly, A. J., and 1ahn R. G. (June 1991). "Anode Power Deposition in an Applied-field Segmented Anode MPD Thrusters," AIAA 91-2343.

[42] Myers, R. M., Kelly, A. J., and Jahn, R. G. (Oct 1998). "Energy Deposition in Low Power Coaxial Plasma Thrusters," IEPC 88-024, presented at 20th International Electric Propulsion Conference, W. Germany.

[43] Myers R. M., Suzuki N., Kelly A. J., and Jahn R. G. (July 1988). "Cathode Phenomena in a Low Power, Steady State MPD Thruster," AIAA - 88-3206.

[44] Auweter-Kurtz, M., and Messerschmid, E. (July 1990). "Plasma Accelerator Activities at the IRS," AIA.A 902659, 21st Intern. Electric Propulsion Conference. 